\title{
Strongly hydrogen-bonded Schiff base and adjoining polyene twisting in the retinal chromophore of schizorhodopsins
}

Tomomi Shionoya, ${ }^{\dagger}$ Manish Singh, ${ }^{\ddagger}$ Misao Mizuno, ${ }^{\dagger}$ Hideki Kandori, ${ }^{\ddagger}, \S$ and Yasuhisa Mizutani ${ }^{\dagger}, *$

† Department of Chemistry, Graduate School of Science, Osaka University, 1-1 Machikaneyama, Toyonaka, Osaka 560-0043, Japan, ‡ Department of Life Science and Applied Chemistry, Nagoya Institute of Technology, Showa-ku, Nagoya, Aichi 466-8555, Japan, and §OptoBio Technology Research Center, Nagoya Institute of Technology, Showa-ku, Nagoya, Aichi 466-8555, Japan. 


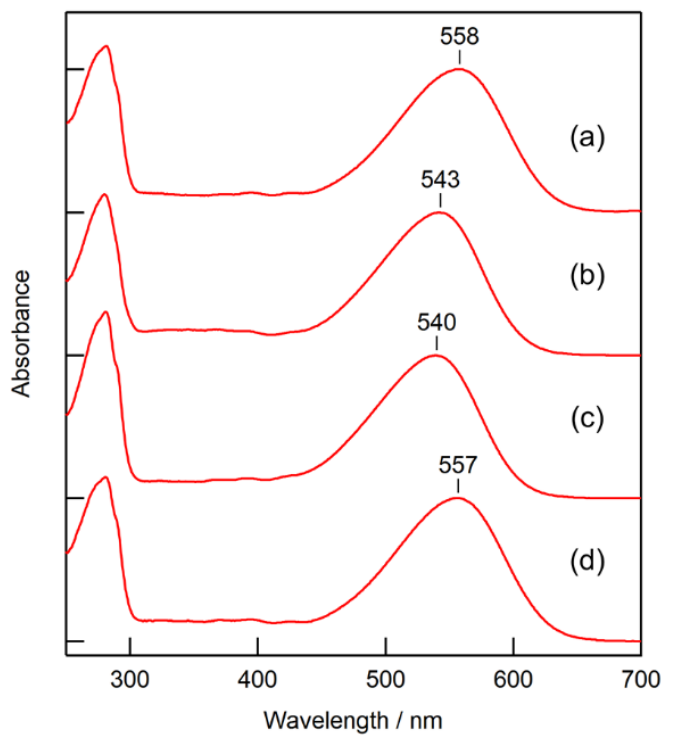

Figure S1. Absorption spectra of SzRs; (a) SzR1, (b) SzR2, (c) SzR3 and (d) SzR4, whose optical purity ratios $\left(A_{280} / A_{\text {chromophore }}\right)$ were $1.17,1.12,1.30$, and 1.15 , respectively. 


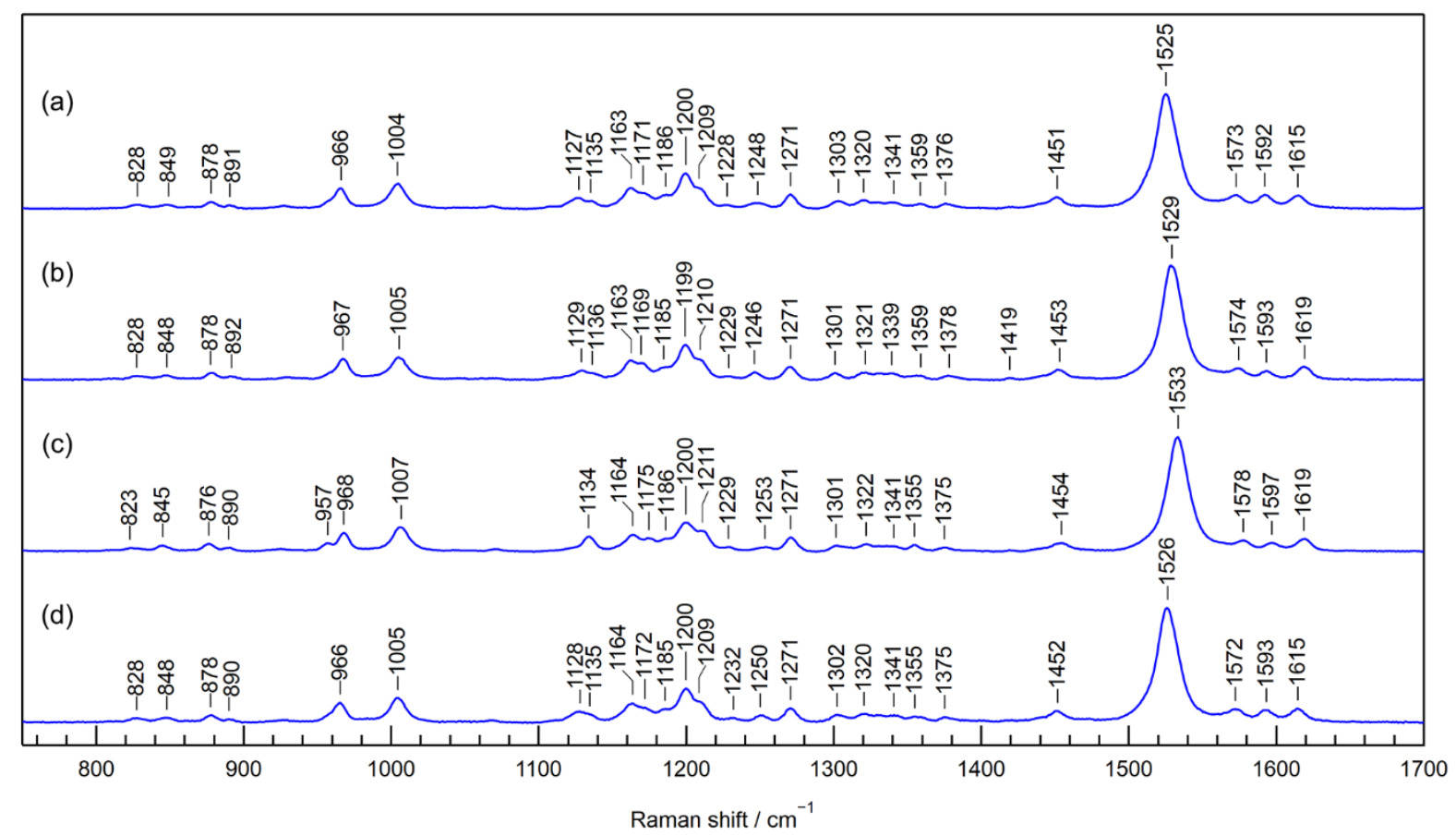

Figure S2. Resonance Raman spectra of SzRs in $\mathrm{D}_{2} \mathrm{O}$ buffer probed at $532 \mathrm{~nm}$. (a) SzR1, (b) SzR2, (c) SzR3 and (d) SzR4. The emission background and the spectral contribution of the buffer were subtracted from each spectrum. 


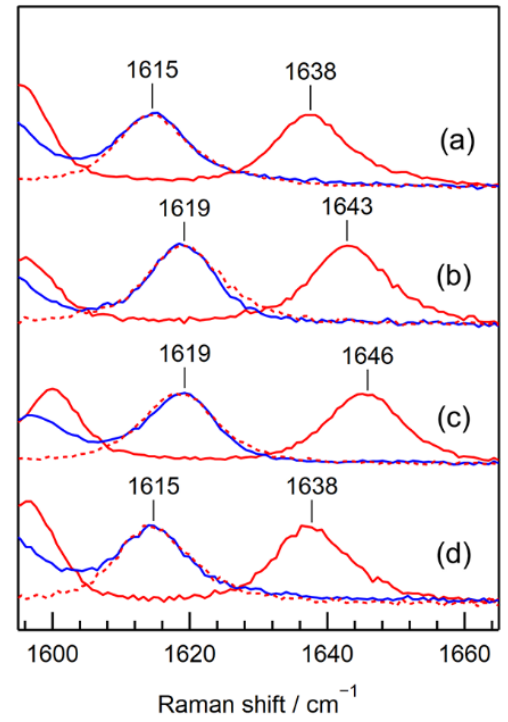

Figure S3. Enlarged views of the resonance Raman spectra of SzRs in the $\mathrm{C}=\mathrm{N}$ stretch region (1560-1680 $\mathrm{cm}^{-1}$ ). (a) SzR1, (b) SzR2, (c) SzR3 and (d) SzR4. Red traces represent the spectra measured in $\mathrm{H}_{2} \mathrm{O}$ buffer. Red dashed traces are the spectra shifted to overlap the $\mathrm{C}=\mathrm{N}$ stretch band in $\mathrm{H}_{2} \mathrm{O}$ buffer onto that measured in $\mathrm{D}_{2} \mathrm{O}$ buffer shown as a blue trace for each sample. 


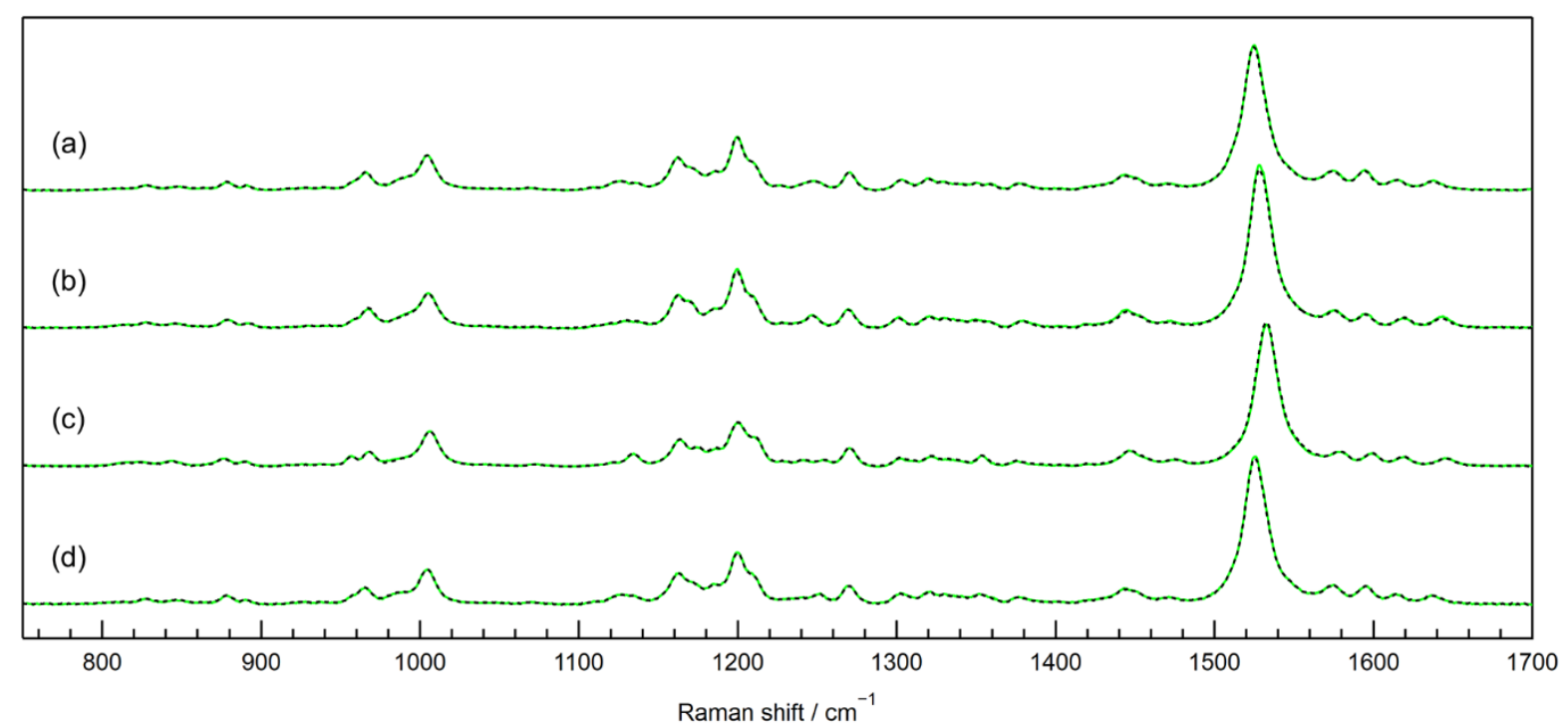

Figure S4. Resonance Raman spectra of SzRs in a mixed buffer $\left(\mathrm{H}_{2} \mathrm{O}: \mathrm{D}_{2} \mathrm{O}=1: 1\right)$ probed at 532 nm. (a) SzR1, (b) SzR2, (c) SzR3 and (d) SzR4. The emission background and the spectral contribution of the buffer were subtracted from each spectrum. Green traces are the spectra measured in the mixed buffer. Black dotted traces are the spectra obtained by calculating the sum of the spectra measured in $\mathrm{H}_{2} \mathrm{O}$ and $\mathrm{D}_{2} \mathrm{O}$ buffer divided by 2 . Band intensities were normalized using the intensity of the methyl rock band appearing around $1005 \mathrm{~cm}^{-1}$ for each spectrum. 


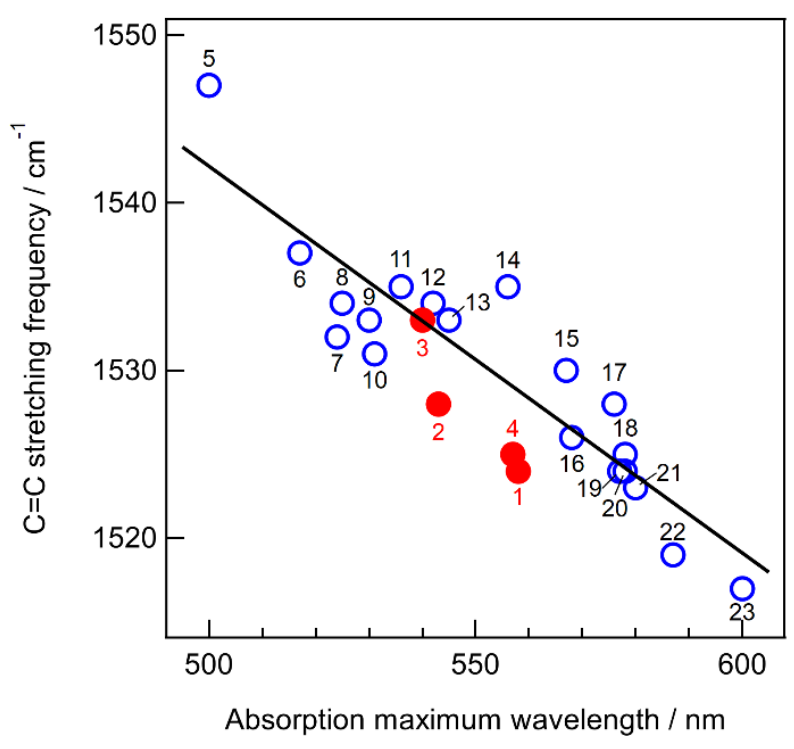

Figure S5. Plot of the $\mathrm{C}=\mathrm{C}$ stretching frequencies against the absorption maximum wavelengths for the retinal chromophore for SzRs in the present study (red circles; 1: SzR1, 2: SzR2, 3: SzR3 and 4: SzR4) and 19 datasets for type-1 rhodopsins reported in previous resonance Raman studies (blue circles; 5: Natronomonas pharaonis SRII; ${ }^{1}$ 6: cation-unbound Krokinobactor rhodopsin 2 (KR2);2 7: K+-bound KR2;2 8: Indibacter alkaliphilus sodium-ion-pump rhodopsin; ${ }^{3}$ 9: thermophilic rhodopsin; 4 10: $\mathrm{Na}^{+}$-bound $\mathrm{KR} 2 ;^{2}$ 11: $\mathrm{Cl}^{-}$-bound Synechocystis halorhodopsin $\left(\right.$ SyHR);5 12: anion-depleted SyHR; ${ }^{5}$ 13: proteorhodopsin; ${ }^{6}$ 14: $\mathrm{SO}_{4}{ }^{2-}$-bound $S y H R ; 5$ 15: $\mathrm{NO}_{3}{ }^{-}$-bound Halobacterium salinarum halorhodopsin $(\mathrm{HsHR}){ }^{7}{ }^{76}$ : Halobacterium salinarum bacteriorhodopsin;8 17: $\mathrm{Cl}^{-}$-bound $\mathrm{HsHR}^{7}$ 18, 19: Cl--bound Natronomonas pharaonis halorhodopsin $(N p H R) ;{ }^{9-10}$ 20: Br--bound $N p H R ; 10$ 21: I--bound $N p H R ;{ }^{10}$ 22: Halobacterium salinarum sensory rhodopsin I; ${ }^{11}$ and 23: anion-depleted $N p \mathrm{HR}^{9}$ ). The black line is the best-fit linear function for the 19 datasets for type- 1 rhodopsins. 


\section{References}

1. Gellini, C.; Lüttenberg, B.; Sydor, J.; Engelhard, M.; Hildebrandt, P., Resonance Raman Spectroscopy of Sensory Rhodopsin II from Natronobacterium pharaonis. FEBS Lett. 2000, 472, 263-266.

2. Otomo, A.; Mizuno, M.; Inoue, K.; Kandori, H.; Mizutani, Y., Allosteric Communication with the Retinal Chromophore Upon Ion Binding in a Light-Driven Sodium Ion-Pumping Rhodopsin. Biochemistry 2020, 59, 520-529.

3. Kajimoto, K.; Kikukawa, T.; Nakashima, H.; Yamaryo, H.; Saito, Y.; Fujisawa, T.; Demura, M.; Unno, M., Transient Resonance Raman Spectroscopy of a Light-Driven Sodium-Ion-Pump Rhodopsin from Indibacter alkaliphilus. J. Phys. Chem. B 2017, 121, 4431-4437.

4. Shionoya, T.; Mizuno, M.; Tsukamoto, T.; Ikeda, K.; Seki, H.; Kojima, K.; Shibata, M.; Kawamura, I.; Sudo, Y.; Mizutani, Y., High Thermal Stability of Oligomeric Assemblies of Thermophilic Rhodopsin in a Lipid Environment. J. Phys. Chem. B 2018, 122, 6945-6953.

5. $\quad$ Niho, A.; Yoshizawa, S.; Tsukamoto, T.; Kurihara, M.; Tahara, S.; Nakajima, Y.; Mizuno, M.; Kuramochi, H.; Tahara, T.; Mizutani, Y.; Sudo, Y., Demonstration of a Light-Driven $\mathrm{SO}_{4}{ }^{2-}$ Transporter and Its Spectroscopic Characteristics. J. Am. Chem. Soc. 2017, 139, 4376-4389.

6. Krebs, R. A.; Dunmire, D.; Partha, R.; Braiman, M. S., Resonance Raman Characterization of Proteorhodopsin's Chromophore Environment. J. Phys. Chem. B 2003, 107, 7877-7883.

7. Maeda, A.; Ogurusu, T.; Yoshizawa, T.; Kitagawa, T., Resonance Raman Study on Binding of Chloride to the Chromophore of Halorhodopsin. Biochemistry 1985, 24, 2517-2521.

8. Smith, S. O.; Lugtenburg, J.; Mathies, R. A., Determination of Retinal Chromophore Structure in Bacteriorhodopsin with Resonance Raman Spectroscopy. J. Membrane Biol. 1985, 85, 95-109.

9. Gerscher, S.; Mylrajan, M.; Hildebrandt, P.; Baron, M.-H.; Müller, R.; Engelhard, M., Chromohore-Anion Interaction in Halorhodopsin from Natronobacterium pharaonis Probed by Time-Resolved Resonance Raman Spectroscopy. Biochemistry 1997, 36, 11012-11020.

10. Mizuno, M.; Nakajima, A.; Kandori, H.; Mizutani, Y., Structural Evolution of a Retinal Chromophore in the Photocycle of Halorhodopsin from Natronobacterium pharaonis. J. Phys. Chem. A 2018, 122, 2411-2423.

11. Fodor, S. P. A.; Gebhard, R.; Lugtenburg, J.; Bogomolni, R. A.; Mathies, R. A., Structure of the Retinal Chromophore in Sensory Rhodopsin-I from Resonance Raman-Spectroscopy. J. Biol. Chem. 1989, 264, 18280-18283. 